\title{
On Modal Components of the $\mathbf{S} 4$-logics
}

\author{
Alexei Y. Muravitsky \\ Louisiana Scholars' College \\ Northwestern State University \\ Natchitoches, LA 71497, U.S.A. \\ alexeim@nsula.edu
}

\begin{abstract}
We consider the representation of each extension of the modal logic S4 as sum of two components. The first component in such a representation is always included in Grzegorczyk logic and hence contains "modal resources" of the logic in question, while the second one uses essentially the resources of a corresponding intermediate logic. We prove some results towards the conjecture that every S4-logic has a representation with the least component of the first kind.
\end{abstract}

\section{Preliminaries}

We consider the intuitionistic propositional logic Int and modal propositional logic $\mathbf{S} 4$, both defined with the postulated rule of substitution, along with the lattices of their normal consistent extensions, NExtInt (intermediate logics) and NExtS4 (S4-logics), respectively. The lattice operations are the set intersection $\cap$ as meet and the deduction closure $\oplus$ as joint. Other logics from NExtInt and NExt $\mathbf{S} \mathbf{4}$ will also appear in the sequel.

The mappings $\rho: N E x t \mathbf{S} \mathbf{4} \longrightarrow N E x t$ Int and $\tau: N E x t$ Int $\longrightarrow N E x t \mathbf{S} \mathbf{4}$ were defined in $[3$. It is well known that the former mapping is a lattice epimorphism and the latter is an embedding; see 3. Another mapping, $\sigma: N E x t \mathbf{I n t} \longrightarrow N E x t \mathbf{G r z}$, defined by the equality $\sigma L=\operatorname{Grz} \oplus \tau L$ for any $L \in N E x t \mathbf{I n t}$, where $\mathbf{G r z}$ is Grzegorczyk logic, is an isomorphism; see [1] and [2]. This, along with inequalities obtained in [3], implies that

$$
\tau \rho M \subseteq M \subseteq \mathbf{G r z} \oplus \tau \rho M,
$$

(Blok-Esakia inequality)

for any logic $M \in N E x t \mathbf{S} 4$.

Thus it can be suggested that any $M \in N E x t \mathbf{S} 4$ is equal to $M^{*} \oplus \tau \rho M$ for some logic $M^{*} \subseteq$ Grz. Indeed, for $M^{*}$ one can always take $M \cap \mathbf{G r z}$; see [4. Furthermore, we have the following.

Let $\mathcal{L}=\{\tau L \mid L \in N E x t$ Int $\}$. An unspecified element of $\mathcal{L}$ will be denoted by $\tau$. Then any $M \in N E x t \mathbf{S 4}$ can be represented as $M=M^{*} \oplus \tau$, where $M^{*} \subseteq \mathbf{G r z}$, i.e. $\rho M^{*}=\mathbf{I n t}$. In this representation of $M$, the first term, $M^{*}$, is called the modal component of $M$ and the second term, $\tau$, is its assertoric (or superintuitionistic) component (or $\tau$-component). Such a representation of $M$ we call a $\tau$-representation.

It has been noticed 4 that the assertoric component of $M$ is uniquely determined by $M$ and equals $\tau \rho M$, but its modal component may vary. Given an S4-logic $M$, the modal components of $M$ constitute a dense sublattice of $N E x t \mathbf{S} 4$ with the top element $M \cap \mathbf{G r z}$. This on-going research aims at proving the conjecture: Every $\mathbf{S} 4$-logic has a least modal component.

\section{Examples of the modal components of some S4-logics}

Below one can see different situations related to modal components of some $\mathbf{S} 4$-logics.

N. Galatos, A. Kurz, C. Tsinakis (eds.), TACL 2013 (EPiC Series, vol. 25), pp. 163-166 
- Each logic in $[\mathbf{S 4}, \mathbf{G r z}]$ itself is its only modal component.

- All $\operatorname{logics} \tau \in \mathcal{L}$ have $\mathbf{S} 4$ their only modal component.

- If $\mathbf{G r z} \subseteq \mathbf{S} 4.1 \oplus \tau$ then the logics of $[\mathbf{S} 4.1, \mathbf{G r z}]$ constitute all the modal components of Grz $\oplus \tau$.

In the sequel we obtain more examples.

\section{$3 \quad S$-series slicing of $N E x t \mathrm{~S} 4$}

We arrange the Scroggs logics [5] as follows:

$$
\mathbf{S 5}=S_{0} \subset \ldots \subset S_{2} \subset S_{1}=\mathbf{S} 4+p \rightarrow \square p .
$$

Definition 3.1 ( $S$-series slicing). A logic $M$ belongs to the $n$th $S$-slice, $n \geq 1$, if $M \subseteq S_{n}$ and $M \nsubseteq S_{n+1}$. If $M \subseteq S_{n}$, for all $n \geq 1$, that is to say, $M \in\left[\mathbf{S 4}, S_{0}\right]$, then $M$ lies in the 0 th $S$-slice. We denote the $n$th $S$-slice by $\mathscr{S}_{n}, n \geq 0$.

Thus $\mathscr{S}_{0}=[\mathbf{S} 4, \mathbf{S 5}]$. Also, it is obvious that $\left\{\mathscr{S}_{n}\right\}_{n \geqslant 0}$ is a partition of NExtS4. As well known, $S_{n}, n \geqslant 1$, is the logic of an $n$-atomic finite interior algebra with only two open elements. We denote such an algebra by $\mathrm{B}_{n}$.

Definition $3.2\left(\operatorname{logics} K_{n}\right)$. Let $\chi_{n}$ be the characteristic formula of algebra $\mathrm{B}_{n}, n \geq 1$. We define $K_{n}=\mathbf{S} 4+\square \chi_{n+1}$, for $n>0$, and $K_{0}=\mathbf{S} 4$.

Proposition 3.1. Each $S$-slice is an interval. For $n \geq 1$, logic $S_{n}$ is the top of the $\mathrm{n}$ th slice and logic $K_{n}$ is its bottom. In particular, $\mathscr{S}_{1}=\left[\mathbf{S} 4.1, S_{1}\right]$.

Corollary 3.1.1. For each $n \geq 1,\left(K_{n}, S_{n+1}\right)$ is a splitting pair in NExtS4.

Proposition 3.2. Let $\tau \in \mathcal{L}$. All logics from the $n$th $S$-slice having $\tau$ as their $\tau$-component constitute the interval $\left[K_{n} \oplus \tau, M_{n} \oplus \tau\right]$.

In addition, we prove the following:

- $K_{n+1} \subset K_{n}$ for any $n \geq 1$; and

- $\bigcap_{n \geqslant 1} K_{n}=\mathbf{S} 4$.

\section{$4 \quad M$-series slicing of $[\mathrm{S} 4, \mathrm{Grz}]$}

We will be using the following notation:

$$
M_{0}=\mathbf{G r z} \cap \mathbf{S} \mathbf{5} \text { and } M_{1}=\mathbf{G r z} .
$$

We note [4 that the interval $\left[M_{0}, M_{1}\right]$ is ordered by $\subset$ in type $1+\omega^{*}$ :

$$
M_{0} \subset \ldots \subset M_{2} \subset M_{1},
$$

where $M_{n}=M_{1} \cap S_{n}$ and $\bigcap_{n \geqslant 1} M_{n}=M_{0}$. To this, we add the following:

- $M_{n} \cap S_{l}$, whenever $M_{l} \subseteq M_{n}$ or $S_{l} \subseteq S_{n}$;

- $K_{n} \oplus S_{l}=S_{n}$, whenever $S_{l} \subseteq S_{n}$; 
- $K_{n} \oplus M_{l}=M_{n}$, whenever $M_{l} \subseteq M_{n}$;

- $M_{n} \cap S_{n+1}=M_{n+1}$, for any $n \geqslant 1$.

Definition 4.1 ( $M$-series slicing). A logic $M$ from $[\mathbf{S 4}, \mathbf{G r z}]$ belongs to the $n$th $M$-slice if and only if $M$ is in the $n$th $S$-slice. In other words, the $n$th $M$-slice equals $\left[K_{n}, S_{n}\right] \cap[\mathbf{S 4}, \mathbf{G r z}] . W e$ denote the $n$th $M$-slice, $n \geq 0$, by $\mathscr{E}_{n}$.

We prove that for any $M \in[\mathbf{S 4}, \mathbf{G r z}]$ and $n \geq 0$, the following conditions are equivalent:

$$
\begin{array}{ll}
\text { a) } & M \in \mathscr{E}_{n} ; \\
\text { b) } & M \in\left[K_{n}, M_{n}\right] ; \\
\text { c) } & M \oplus M_{0}=M_{n} .
\end{array}
$$

For any $n \geqslant 1$, each of $(a)-(c)$ is equivalent to:

$$
\text { d) } M \subseteq M_{n} \text { and } M \nsubseteq M_{n+1} \text {. }
$$

Proposition 4.1. Let us fix $n \geqslant 0$. If a modal logic $M$ lies in $\mathscr{S}_{n}$, then any its modal component $M^{*}$ belongs to $\mathscr{E}_{n}$. Conversely, for any modal logic $M^{*}$ in $\mathscr{E}_{n}$ and any $\tau$ in $\mathcal{L}$, the logic $M^{*} \oplus \tau$ lies in $\mathscr{S}_{n}$.

From Proposition 4.1 and some properties mentioned above we derive:

- For any $n \geqslant 0$, all modal logics of $\mathscr{E}_{n}$ are the modal components of the logic $S_{n}$.

Also, we obtain the following: Given $\tau \in \mathcal{L}$,

- if $M_{n} \subseteq K_{n} \oplus \tau$ then the logics of $\left[K_{n}, M_{n}\right]$ constitute all the modal components of $M_{n} \oplus \tau$;

\section{Least modal components}

In this section we will show that the existence of the least modal component of a logic $M$ can be reduced to the question of definability of some function of $M$. The proposition of this section states that the definability of this function should be checked for some logics of the 0th S-slice.

Definition 5.1 (Mappings $h_{n}, h_{n 0}$, and $g_{n 0}$ ). For any $n \geqslant 0$, we define: $h_{n}: M \mapsto M \cap S_{n}$, where $M \in N E x t \mathbf{S} 4$. We denote by $h_{n 0}$ and by $g_{n 0}$ the mapping $h_{0}$ restricted to $\mathscr{S}_{n}$ and $\mathscr{E}_{n}$, respectively.

We observe the following:

- $h_{n 0}$ a lattice embedding of $\mathscr{S}_{n}$ into $\mathscr{S}_{0}$;

- $g_{n 0}$ is a lattice embedding of $\mathscr{E}_{n}$ into $\mathscr{E}$.

Proposition 5.1. Given an S4-logic $M \in \mathscr{S}_{n}, M$ has a least modal component if and only if $h_{n 0}(M)$ has it.

Definition 5.2 (difference operation $d(X, Y)$ ). Given two logics $X$ and $Y$, a logic $C \subseteq X$ is called the difference of the subtraction of $Y$ from $X$, if for any logic $Z$, the following equivalence holds:

$$
C \subseteq Z \subseteq X \Leftrightarrow X=Z \oplus Y .
$$

If such $C$ exists for given $X$ and $Y$, it is obviously unique. We denote it by $d(X, Y)$. 
The operation $d(X, Y)$ is certainly partial. For instance, $d\left(M_{0}, \mathbf{D u m}\right)$ is undefined, where $\mathbf{D u m}=\mathbf{S} 4+\square(\square(p \rightarrow \square p) \rightarrow p) \rightarrow(\diamond \square p \rightarrow p)$.

Next we define: Given $M \in N E x t \mathbf{S} 4$,

$$
d^{*}(M)=d(M, \tau \rho M) .
$$

Proposition 5.2. Given $M \in N E x t \mathbf{S 4}, d^{*}(M)$ is defined if and only if $d^{*}(M)$ is the least modal component of $M$.

The next theorem shows that our search for the definability of the $d^{*}$ function on NExtS4 can be reduces to the 0 th $\mathrm{S}$-slice.

Proposition 5.3. Every $\mathbf{S} 4$-logic has its least modal component if and only if for any $M^{*} \in$ $\left[\mathbf{S} 4, M_{0}\right]$ and $\tau \in \mathcal{L}, d\left(M^{*}, \tau \cap M^{*}\right)$ is defined, or, equivalently, $d^{*}\left(M^{*} \oplus \tau\right)$ is defined, providing that $\tau \cap \mathbf{G r z} \subseteq M^{*}$.

\section{Greatest modal components}

We remind the reader that any logic $M \in N E x t(\mathbf{S} 4)$ has its greatest modal component which is $M \cap \mathbf{G r z}$. Also, we know from Proposition 3.2 that all $\mathbf{S} 4$-logics of the $n$th S-slice that have a $\operatorname{logic} \tau \in \mathcal{L}$ constitute the interval $\left[K_{n} \oplus \tau, M_{n} \oplus \tau\right]$. The next proposition reads that the greatest modal components of the logics of the last set form an interval.

Proposition 6.1. Let $\tau \in \mathcal{L}$. The greatest modal components of all logics from the $n$th $S$-slice having $\tau$ as their $\tau$-component constitute the interval $\left[K_{n} \oplus(\mathbf{G r z} \cap \tau), M_{n}\right]$.

\section{References}

[1] W. J. Blok. Varieties of Interior Algebras. PhD thesis, University of Amsterdam, 1976.

[2] L. L. Esakia. On modal "counterparts" of superintuitionistic logics. In The Seventh All-Union Symposium on Logic and Methodology of Science, Abstracts (Russian), pages 135-136. Kiev, 1976.

[3] L. L. Maksimova and V. V. Rybakov. The lattice of normal modal logics. Algebra i Logika, 13:188216, 235, 1974.

[4] A. Y. Muravitsky. The embedding theorem: its further developments and consequences. Part I. Notre Dame J. Formal Logic, 47(4):525-540, 2006.

[5] S. J. Scroggs. Extensions of the Lewis system S5. J. Symbolic Logic, 16:112-120, 1951. 